\title{
Equivariant Controllable Cutting-Pasting and Cobordism with Vector Fields
}

By

\author{
Katsuhiro KomrYA*
}

\section{Introduction}

Throughout this paper $G$ always denotes a finite group of odd order, and manifolds and maps considered are all of class $C^{\infty}$. $\mathscr{M}_{n}[G]$ denotes the set of $n$-dimensional closed $G$-manifolds. In the set $\mathscr{M}_{n}[G]$ we will consider the two notions, Reinhart $G$-cobordism and $S K K$-equivalence.

For $M$ and $N \in \mathscr{M}_{n}[G]$, if there is an $(n+1)$-dimensional compact $G$-manifold $L$ with $\partial L=M+N$, the disjoint union of $M$ and $N$, then they are called $G$-cobordant and $L$ is called a $G$-cobordism between them. This cobordism relation defines the cobordism group $N_{n}[G]$ of $n$ dimensional closed $G$-manifolds. If a $G$-cobordism $L$ between $M$ and $N$ admits a nonzero $G$-vector field which is inward normal on $M$ and outward normal on $N$, then, following Reinhart [8], $M$ and $N$ are called Reinhart $G$-cobordant, and $L$ a Reinhart $G$-cobordism between them. The set of cobordism classes by this cobordism relation in $\mathscr{M}_{n}[G]$ forms a semigroup with disjoint union + as its group operation. Denote by $N_{n}^{R}[G]$ the Grothendieck group of the semigroup. From the author [4] we obtain a necessary and sufficient condition for $M$ and $N \in \mathscr{M}_{n}[G]$ to represent the same class in $N_{n}^{R}[G]$ in terms of $N_{n}[G]$ and the Euler characteristics of the fixed point sets of $M$ and $N$.

Let $P, P^{\prime}, Q$ and $Q^{\prime}$ be $n$-dimensional compact $G$-manifolds with $\partial P=\partial P^{\prime}$ and $\partial Q=\partial Q^{\prime}$. Let $\varphi$ and $\phi: \partial P \rightarrow \partial Q$ be $G$-diffeomorphisms. Then by pasting two $G$-manifolds along boundary we obtain closed $G$-manifolds $P \cup_{\varphi} Q, P^{\prime} \cup_{\psi} Q^{\prime}$, etc. Give $\mathscr{M}_{n}[G]$ the equivalence relation

Communicated by N. Shimada, June 4, 1986.

*Department of Mathematics, Yamaguchi University, Yamaguchi 753, Japan. 
$\sim$ generated by relations of the form

$$
P \cup_{\varphi} Q+P^{\prime} \cup_{\psi} Q^{\prime} \sim P \cup_{\psi} Q+P^{\prime} \cup_{\varphi} Q^{\prime} .
$$

This relation is called $S K K$-equivalence (Schneiden und Kleben Kontrollierbar). The quotient set $\mathscr{M}_{n}[G] / \sim$ becomes a semigroup with the operation + . Denote by $S K K_{n}[G]$ the Grothendieck group of the semigroup.

In this paper we will establish short exact sequences which contain the groups $N_{n}[G], N_{n}^{R}[G]$ and $S K K_{n}[G]$. From the exact sequences we will show, under a restriction for the one dimensional components of the fixed point sets, $M$ and $N$ represent the same class in $N_{n}^{R}[G]$ if and only if so do they in $S K K_{n}[G]$.

Heithecker $[2,3]$ also discusses $S K K$-equivalence and Reinhart $G$-cobordism of oriented $G$-manifolds, $G$ an abelian group of odd order. A modified version of $S K K$-equivalence is discussed in Prevot $[5,6,7]$.

\section{$\S 1$. Surgery}

Given a $G$-manifold $M$ and a subgroup $H$ of $G, M^{H}$ denotes the $H$-fixed point set of $M$ and $M^{=H}$ denotes the union of those components of $M^{H}$ on which $H$ occurs actually as an isotropy subgroup. If $V$ is a representation of $H$ containing no direct summand of trivial representation, $M^{(H, V)}$ denotes the union of those components of $M^{=H}$ at which the normal representation is isomorphic to $V$. Suppose $M^{(H, V)} \neq \phi$. Denote by $G_{(H, V)}$ the subgroup of $G$ whose action keeps $M^{(H, V)}$ invariant. We see that $H \subset G_{(H, V)} \subset N(H)$, the normalizer of $H$ in $G$, and that $G_{(H, V)}$ is determined by $(H, V)$ and independent of $M$.

Let $\operatorname{dim} M^{(H, V)}>0$ and $\operatorname{dim} M^{(H, V)}+1=p+q$ where $p$ and $q$ are positive integers. Then there is a smooth $G$-embedding $\varphi: G \times{ }_{H}$ $D\left(V \oplus R^{p}\right) \times S\left(R^{q}\right) \rightarrow M$ onto a $G$-invariant regular submanifold of $M$, where $D(), S()$ and $R^{k}$ denote the closed unit disc, the unit sphere and the $k$-dimensional trivial representation, respectively. Let $L$ be a $G$-manifold obtained from the disjoint union of $M \times[0,1]$ and $G \times{ }_{H} D\left(V \oplus R^{p}\right) \times D\left(R^{q}\right)$ by identifying $\operatorname{Im} \varphi \times\{1\}$ with $G \times{ }_{H} D\left(V \oplus R^{p}\right)$ $\times S\left(R^{q}\right)$. Then, if $M$ is closed, $\partial L=M+N$ where

$$
N=\left(M-\varphi\left(G \times{ }_{H} \stackrel{\circ}{D}\left(V \oplus R^{p}\right) \times S\left(R^{q}\right)\right) \cup G \times_{H} S\left(V \oplus R^{p}\right) \times D\left(R^{q}\right),\right.
$$


$\stackrel{D}{D}()$ denotes the open unit disc。 $N$ is called a $G$-manifold obtained from $M$ by $G$-surgery of type $(H, V, p, q)$, and $L$ the trace of the $G$ surgery. We see the following.

(1) If $\operatorname{dim} M^{(K, U)}<\operatorname{dim} M^{(H, V)}$, then the $G$-surgery does not affect $M^{(K, U)}$, i. e., $M^{(K, U)}=N^{(K, U)}$.

(2) If $\operatorname{dim} M^{(H, V)}$ is even, then

$$
\chi\left(N^{(H, V)}\right)=\chi\left(M^{(H, V)}\right)+(-1)^{p+1} 2 \chi\left(G_{(H, V)} / H\right),
$$

where $\chi()$ denotes the Euler characteristic, because $N^{(H, V)}$ is obtained from $M^{(H, V)}$ by deleting $\chi\left(G_{(H, V)} / H\right)$ copies of $\stackrel{D}{D}\left(R^{p}\right) \times S\left(R^{q}\right)$ and attatching as many copies of $S\left(R^{p}\right) \times D\left(R^{q}\right)$.

(3) If $M^{(H, V)}$ is connected and $p>1$, then $N^{(H, V)}$ is also connected. If $\operatorname{dim} M^{(H, V)}$ is even and greater than 2, we may take $p$ to be odd and greater than 1. Thus, by doing $G$-surgeries of an appropriate type we may then obtain $N$ such that $N^{(H, V)}$ is connected and $\chi\left(N^{(H, V)}\right)>0$.

The following lemma is obtained from the existence of excellent $G$-Morse functions (see Field [1]) and a usual connection between surgery and Morse function.

Lemma $\mathbb{1}_{0} \mathbb{1}_{\text {. Let }} M$ and $N \in \mathscr{M}_{n}[G]$ be $G$-cobordant, and $L$ a $G$ cobordism between them. Then $N$ is obtained from $M$ by performing a finite series of $G$-surgeries of type $\left(H_{i}, V_{i}, p_{i}, q_{i}\right), i=1,2, \ldots, s$, with trace $L_{\text {。 }}$

\section{§2. Cobordism with Vector Fields}

Lemma 2.1 ([3; Satz 1.1], [4; Proposition 1.2], [9; Theorem 4.4]). Let $L$ be a $G$-cobordism between closed $G$-manifolds $M$ and $N$. Then $L$ admits a nonzero $G$-vector field which is inward normal on $M$ and outward normal on $N$, if and only if for any subgroup $H$ of $G$, every component $A$ of $M^{H}$ satisfies $\chi(A)=\chi(A \cap M)=\chi(A \cap N)$.

For a space $X$ and a nonnegative integer $k, k X$ denotes the disjoint union of $k$ copies of $X$. For a $G$-manifold $M, M^{H, k}$ denotes the $k$-dimensional components of $M^{H}$. If $M \in \mathscr{M}_{n}[G],[M]$ denotes the class represented by $M$ in the group $N_{n}[G], N_{n}^{R}[G]$ or $S K K_{n}[G]$.

Lemma 2.2. Suppose that $[M]=[N]$ in $N_{n}[G]$, and that $M^{H, 1}=\phi$ and $N^{H, 1}=\phi$ for any subgroup $H$ of $G$. Then there exists a compact $G$ - 
manifold $L$ such that

(i) $\partial L=M_{0}+N_{0}$

$$
\begin{aligned}
& M_{0}=M+\sum_{(H, V)} \alpha_{(H, V)} G \times_{H} S\left(V \oplus R^{n-\operatorname{dim} V+1}\right), \\
& N_{0}=N+\sum_{(H, V)} \beta_{(H, V)} G \times{ }_{H} S\left(V \oplus R^{n-\operatorname{dim} V+1}\right),
\end{aligned}
$$

where $\alpha_{(H, V)}$ and $\beta_{(H, V)}$ are nonnegative integers, and the sums $\sum_{(H, V)}$ are taken over pairs $(H, V)$ of subgroups $H$ of $G$ and representations $V$ of $H$ which contain no direct summand of trivial representation, and

(ii) for any subgroup $H$ of $G$ every component $A$ of $L^{H}$ satisfies $\chi(A)=\chi\left(A \cap M_{0}\right)=\chi\left(A \cap N_{0}\right)$.

From Lemma 2. 1 it follows that the $G$-manifold $L$ in Lemma 2.2 admits a nonzero $G$-vector field which is inward normal on $M_{0}$ and outward normal on $N_{0}$. Thus we see

Proposition 2.3. Suppose that $[M]=[N]$ in $N_{n}[G]$, and that $M^{H, 1}=\phi$ and $N^{H, 1}=\phi$ for any subgroup $H$ of $G$. Then in $N_{n}^{R}[G]$,

$$
[M]=[N]+\sum_{(H, V)} \gamma_{(H, V)}\left[G \times{ }_{H} S\left(V \oplus R^{n-\operatorname{dim} V+1}\right)\right],
$$

where $\gamma_{(H, V)}$ are integers.

Proof of Lemma 2.2. (1) From the hypothesis there exists a compact $G$-manifold $L_{0}$ such that (i) $\partial L_{0}=M+N$, and (ii) $L_{0}^{H, 2}$ is closed for any subgroup $H$. We eliminate all the isolated $H$-fixed points from $L_{0}$ as follows. Take in $L_{0}$ invariant small open discs $\bigcup_{V} D^{\circ}(V)$ with the isolated $H$-fixed points as their centers. Gut the discs off from $L_{0}$, and sew the resulting manifold along the newly arising boundary $\cup_{V} S(V)$ by antipodal involution. Since $G$ is of odd order, no new fixed points arise by this process, and we obtain a compact $G$-manifold $L_{1}$ such that (i) $\partial L_{1}=M+N$, and (ii) for any subgroup $H$ of $G, L_{1}^{H, 0}$ is empty and $L_{1}^{H, 2}$ is closed.

(2) Cut an invariant small open tubular neighborhood of $L_{1}^{H, 2}$ off from $L_{1}$. Then the newly arising boundary is a sphere bundle. Sew the resulting manifold along the new boundary by antipodal involution (on the sphere bundle). Since $G$ is of odd order, no new fixed points arise by this process also. Thus we obtain a compact $G$-manifold $L_{2}$ such that (i) $\partial L_{2}=M+N$, and (ii) $L_{2}^{H, 0}$ and $L_{2}^{H, 2}$ are empty for any subgroup $H$ of $G$. 
(3) If for a component $A$ of $L_{2}^{H, 1}, \partial A \cap M$ or $\partial A \cap N$ is two points, then take a small open $\operatorname{disc} \stackrel{\circ}{D}(V \oplus R)$ with a point $\in \operatorname{Int} A$ as its center. We cut such discs off from $L_{2}$, and then obtain a compact $G$-manifold $L_{3}$ such that

(i)

$$
\begin{aligned}
& \partial L_{3}=M_{3}+N_{3}, \\
& M_{3}=M+\sum \alpha_{(H, V)} G \times_{H} S(V \oplus R), \\
& N_{3}=N+\sum \beta_{(H, V)} G \times_{H} S(V \oplus R),
\end{aligned}
$$

(ii) for any subgroup $H$ of $G$, any component of $L_{3}^{H, 1}$ is either a closed curve $\left(\approx S^{1}\right)$ or a curve in $L_{3}$ which joins a point of $M_{3}$ and a point of $N_{3}$, and

(iii) $L_{3}^{H, 0}$ and $L_{3}^{H, 2}$ are empty for any subgroup $H$ of $G$. Thus $L_{3}$ is a $G$-cobordism between $M_{3}$ and $N_{3}$ such that $\chi(A)=\chi\left(A \cap M_{3}\right)$ $=\chi\left(A \cap N_{3}\right)$ for any component $A$ of $L_{3}^{H, r}, r=0,1,2$.

(4) For a positive integer $k$ consider the following assertion:

$P(k)$. There exists a compact $G$-manifold $L_{k}$ such that

(i) $\partial L_{k}=M_{k}+N_{k}$,

$$
\begin{aligned}
& M_{k}=M+\sum_{(H, V)} \alpha_{(H, V)} G \times{ }_{H} S\left(V \oplus R^{n-\mathrm{dim} V+1}\right), \\
& N_{k}=N+\sum_{(H, V)} \beta_{(H, V)} G \times{ }_{H} S\left(V \oplus R^{n-\mathrm{dim} V+1}\right),
\end{aligned}
$$

where $\alpha_{(H, V)}$ and $\beta_{(H, V)}$ are nonnegative integers, and

(ii) for any subgroup $H$ of $G$ and any component $A$ of $L_{k}^{H, r}(r<k)$,

$$
\chi(A)=\chi\left(A \cap M_{k}\right)=\chi\left(A \cap N_{k}\right) \text { 。 }
$$

If $k \leqq 3$, the assertion $P(k)$ is already proved by the above arguments. We prove below that $P(k)$ implies $P(k+1)$ for $k \geqq 3$. Since Lemma 2. 2 is equivalent to $P(n+2)$, the lemma is inductively obtained.

For a pair $(H, V)$ suppose that $\operatorname{dim} L_{k}^{(H, V)}=k$. It is no loss of generality to suppose that $L_{k}^{(H, V)}$ is connected, since if it is not, we may make it connected by $G$-surgery of type $(H, V, k, 1)$. Moreover, as noted in $\S 1$ this $G$-surgery does not affect $L_{k}^{K, r}$ for any subgroup $K \leqq G$ and any $r<k$. Since $M_{k}^{(H, V)}$ and $N_{k}^{(H, V)}$ are cobordant with a cobordism $L_{k}^{(H, V)}$, then $\chi\left(M_{k}^{(H, V)}\right)-\chi\left(N_{k}^{(H, V)}\right)$ is even. The assertion (ii) of $P(k)$ implies $\chi\left(\left(M_{k}^{(H, V)}\right)^{K}\right)=\chi\left(\left(N_{k}^{(H, V)}\right)^{K}\right)$ for any subgroup $K$ with $H \leqq K \leqq G_{(H, V)}$. From this we see that $\chi\left(M_{k}^{(H, V)}\right)-\chi\left(N_{k}^{(H, V)}\right)$ is 
a multiple of $\chi\left(G_{(H, V)} / H\right)$, since $H$ is the principal isotropy subgroup of the $G_{(H, V)}$-manifolds $M_{k}^{(H, V)}$ and $N_{k}^{(H, V)}$. Thus we may put

$$
\chi\left(M_{k}^{(H, V)}\right)-\chi\left(N_{k}^{(H, V)}\right)=2 m \chi\left(G_{(H, V)} / H\right)
$$

for some integer $m$.

(5) Suppose that $k=\operatorname{dim} L_{k}^{(H, V)}$ is odd. It then follows that

$$
\begin{aligned}
\chi\left(L_{k}^{(H, V)}\right) & =\frac{1}{2} \chi\left(\partial L_{k}^{(H, V)}\right) \\
& =\frac{1}{2}\left(\chi\left(M_{k}^{(H, V)}\right)+\chi\left(N_{k}^{(H, V)}\right)\right) \\
& =\chi\left(M_{k}^{(H, V)}\right)-m \chi\left(G_{(H, V)} / H\right) \\
& =\chi\left(N_{k}^{(H, V)}\right)+m \chi\left(G_{(H, V)} / H\right) .
\end{aligned}
$$

Take $|m|$ points $x_{1}, x_{2}, \ldots, x_{|m|}$ of Int $L_{k}^{(H, V)}$ whose isotropy subgroups are all $H$ and for which $g x_{i} \neq x_{j}$ if $g \in G_{(H, V)}$ and $i \neq j$. Consider a small disc $D_{i}\left(V \oplus R^{k}\right)$ with $x_{i}$ as its center, and let

$$
L_{k}^{\prime}=L_{k}-\cup_{i=1}^{|m|} G \times{ }_{H}{\stackrel{\circ}{D_{i}}}_{(}\left(V \oplus R^{k}\right) .
$$

We then see that

$$
\partial L_{k}^{\prime}=M_{k}+N_{k}+|m| G \times_{H} S\left(V \oplus R^{k}\right) .
$$

If $m \geqq 0$, then let

$$
\begin{aligned}
& M_{k}^{\prime}=M_{k}, \quad \text { and } \\
& N_{k}^{\prime}=N_{k}+|m| G \times_{H} S\left(V \oplus R^{k}\right) .
\end{aligned}
$$

If $m<0$, then let

$$
\begin{aligned}
& M_{k}^{\prime}=M_{k}+|m| G \times_{H} S\left(V \oplus R^{k}\right), \text { and } \\
& N_{k}^{\prime}=N_{k} .
\end{aligned}
$$

$L_{k}^{\prime(H, V)}$ is then connected and satisfies $\chi\left(L_{k}^{\prime(H, V)}\right)=\chi\left(M_{k}^{\prime(H, V)}\right)=\chi\left(N_{k}^{\prime(H, V)}\right)$. Thus, performing the same as above for all $(H, V)$ with $\operatorname{dim} L_{k}^{(H, V)}=k$, we obtain a compact $G$-manifold $L_{k+1}$ as in $P(k+1)$.

(6) Suppose that $k=\operatorname{dim} L_{k}^{(H, V)}$ is even. Since $\chi\left(M_{k}^{(H, V)}\right)=0$ and $\chi\left(N_{k}^{(H, V)}\right)=0$, we must then make the Euler characteristic of $L_{k}^{(H, V)}$ zero (keeping the connectedness of $\left.L_{k}^{(H, V)}\right)$. The assertion (ii) of $P(k)$ implies $\chi\left(\left(L_{k}^{(H, V)}\right)^{K}\right)=0$ for any subgroup $K$ with $H \leqq K \leqq G_{(H, V)}$. From this we see that $\chi\left(L_{k}^{(H, V)}\right)$ is a multiple of $\chi\left(G_{(H, V)} / H\right)$. Thus let $\chi\left(L_{k}^{(H, V)}\right)=m \chi\left(G_{(H, V)} / H\right), m$ an integer. We may suppose that $m$ is nonnegative, since if it is not, we may make $\chi\left(L_{k}^{(H, V)}\right)$ nonnegative 
by the argument (3) in $\S 1$. Take $m$ points $x_{1}, x_{2}, \ldots, x_{m}$ of $\operatorname{Int} L_{k}^{(H, V)}$ whose isotropy subgroups are all $H$ and for which $g x_{i} \neq x_{j}$ if $g \in G_{(H, V)}$ and $i \neq j$. Consider a small disc $D_{i}\left(V \oplus R^{k}\right)$ with $x_{i}$ as its center, and let

$$
L_{k}^{\prime}=L_{k}-\bigcup_{i=1}^{m} G \times{ }_{H} \stackrel{\circ}{D_{i}}\left(V \oplus R^{k}\right) .
$$

We then see that

$$
\partial L_{k}^{\prime}=M_{k}+N_{k}+m G \times{ }_{H} S\left(V \oplus R^{k}\right) 。
$$

Letting $M_{k}^{\prime}=M_{k}$ and $N_{k}^{\prime}=N_{k}+m G \times{ }_{H} S\left(V \oplus R^{k}\right)$, we see that $L_{k}^{\prime(H, V)}$ is connected and $\chi\left(L_{k}^{\prime(H, V)}\right)=\chi\left(M_{k}^{\prime(H, V)}\right)=\chi\left(N_{k}^{\prime(H, V)}\right)=0$. Thus, performing the same as above for all $(H, V)$ with $\operatorname{dim} L_{k}^{(H, V)}=k$, we obtain a compact $G$-manifold $L_{k+1}$ as in $P(k+1)$.

Let $I_{n}^{R}[G]$ be the subgroup of $N_{n}^{R}[G]$ generated by $G$-manifolds of the form $G \times{ }_{H} S(V)$ where $H$ is any subgroup of $G$, and $V$ is any representation of $H$ with $\operatorname{dim} V=\mathrm{n}+1$. Let $\bar{N}_{n}[G]$ and $\bar{N}_{n}^{R}[G]$ be the subgroups of $N_{n}[G]$ and $N_{n}^{R}[G]$, respectively, generated by $G-$ manifolds $M$ with $M^{H, 1}=\phi$ for any subgroup $H$ of $G$. Let $\bar{I}_{n}^{R}[G]$ $=I_{n}^{R}[G] \cap \bar{N}_{n}^{R}[G]$. If a representation $V$ of an odd order group contains no direct summand of trivial representation, $V$ has a complex structure, and hence its (real) dimension is even. This implies that the dimensions of $M$ and its fixed point sets are congruent modulo 2. Thus if $\operatorname{dim} M$ is even, $M^{H, 1}$ is always empty. If $G$ is abelian, the normal bundle of $M^{H, 1}$ in $M$ are Reinhart $G$-cobordant to zero as $G$-vector bundle (see the author [4; Lemma 5. 1]). Thus if $n$ is even or if $G$ is abelian, then we see that $N_{n}[G]=\bar{N}_{n}[G], \quad N_{n}^{R}[G]=\bar{N}_{n}^{R}[G]$ and $I_{n}^{R}[G]=\bar{I}_{n}^{R}[G]$.

Theorem 2.4. There is a short exact sequence

$$
0 \longrightarrow \bar{I}_{n}^{R}[G] \stackrel{i}{\longrightarrow} \bar{N}_{n}^{R}[G] \stackrel{j}{\longrightarrow} \bar{N}_{n}[G] \longrightarrow 0
$$

where $i$ is the canonical inclusion, and $j$ is the obvious homomorphism, $i_{0} e_{\text {., }}$ the homomorphism sending a Reinhart G-cobordism class $[M]$ to a $G$-cobordism class $[M]$.

Proof. It is easy that $i$ is monic, $j$ is epic and $j \circ i=0$. Ker $j \subset \operatorname{Im}$ $i$ follows from Proposition 2.3. 
Proposition 2.5. If $n$ is odd, then $I_{n}^{R}[G]=0$.

Proof. It is sufficient to prove that $\left[G \times{ }_{H} S(U)\right]=0$ in $I_{n}^{R}[G]$ if $U$ is a representation of $H$ with $\operatorname{dim} U=n+1$ even. Note that $\operatorname{dim} U^{K}$ is even for any subgroup $K$ of $H$. Let $R P(U \oplus R)$ be the quotient space $S(U \oplus R)$ by the antipodal involution. It inherits a stucture of an $n$-dimensional $H$-manifold. In $R P(U \oplus R)$ take a small disc $D(U)$ with the point $R P(R)$ as its center, and let

$$
L=G \times{ }_{H} R P(U \oplus R)-G \times{ }_{H} \stackrel{\circ}{ }(U) .
$$

We then see that $\partial L=G \times{ }_{H} S(U)$ and that for any subgroup $K$ of $G$ any component $A$ of $L^{K}$ is diffeomorphic to $R P\left(U^{\prime} \oplus R\right)-\stackrel{\circ}{D}\left(U^{\prime}\right)$ (where $U^{\prime}$ is an even dimensional subspace of $U$ ) and $A$ satisfies $\chi(A)=\chi(\partial A)=0$. By Lemma 2.1 $L$ admits a nonzero $G$-vector field which is inward normal on $\partial L$. Thus $\left[G \times{ }_{H} S(U)\right]=0$.

Note that any element of the Grothendieck group of a semigroup $S$ is of the form $s-s^{\prime}$ where $s, s^{\prime} \in S$.

Proposition 2.6. Suppose that $[M]-[N]$ is an element of $I_{n}^{R}[G]$. Then $[M]-[N]=0$ in $I_{n}^{R}[G]$ if and only if $\chi\left(M^{(H, V)}\right)=\chi\left(N^{(H, V)}\right)$ for any pair $(H, V)$.

Proof. $[M]-[N]=0$ implies $\chi\left(M^{(H, V)}\right)=\chi\left(N^{(H, V)}\right)$ by Lemma 2.1. If $n$ is odd, $[M]-[N]=0$ is clear by the preceding proposition. Suppose that $n$ is even and that $\chi\left(M^{(H, V)}\right)=\chi\left(N^{(H, V)}\right)$ for any $(H, V)$. Note that in this case the dimensions of fixed point sets are all even. It is sufficient to prove that $[M]-[N]=0$ when

$$
\begin{aligned}
& M=\sum_{(H, V) \in T(G)} \alpha_{(H, V)} G \times_{H} S\left(V \oplus R^{n-\operatorname{dim} V+1}\right), \text { and } \\
& N=\sum_{(H, V) \in T(G)} \beta_{(H, V)} G \times_{H} S\left(V \oplus R^{n-\operatorname{dim} V+1}\right)
\end{aligned}
$$

Here $\alpha_{(H, V)}$ and $\beta_{(H, V)}$ are nonnegative integers, and $T(G)$ is a finite set of pairs $(H, V)$ such that if $(H, V) \neq(K, U)$ in $T(G)$, then $G \times{ }_{H} S\left(V \oplus R^{n-\operatorname{dim} V+1}\right)$ and $G \times{ }_{K} S\left(U \oplus R^{n-\operatorname{dim} U+1}\right)$ are not $G$-diffeomorphic. Order the pairs in

$$
T(G)=\left\{\left(H_{1}, V_{1}\right),\left(H_{2}, V_{2}\right), \ldots,\left(H_{a}, V_{a}\right)\right\}
$$

in such a way that if $H_{i}$ is conjugate to a subgroup of $H_{j}$, then $j \leqq i$. 
It follows that

$$
\left(G \times_{H_{j}} S\left(V_{j} \oplus R^{n-\operatorname{dim} V_{j}+1}\right)\right)^{\left(H_{i}, V_{i}\right)}= \begin{cases}G_{\left(H_{i}, V_{i}\right)} / H_{i} \times S\left(R^{n-\operatorname{dim} V_{i}+1}\right) & \text { if } i=j \\ \phi & \text { if } i<j .\end{cases}
$$

Thus we see that $\chi\left(M^{\left(H_{i}, V_{i}\right)}\right)=\sum_{j=1}^{i} \varepsilon_{j} \alpha_{\left(H_{j}, V_{j}\right)}$ and $\chi\left(N^{\left(H_{i}, V_{i}\right)}\right)=\sum_{j=1}^{i} \varepsilon_{j} \beta_{\left(H_{j}, V_{j}\right) \text { g }}$ where $\varepsilon_{j}$ is an integer, especially $\left.\varepsilon_{i}=2 \chi\left(G_{\left(H_{i}, V_{i}\right)}\right) H_{i}\right) \neq 0$. Since $\chi\left(M^{\left(H_{i}, V_{i}\right)}\right)$ $=\chi\left(N^{\left(H_{i}, V_{i}\right)}\right)$, then $\alpha_{\left(H_{i}, V_{i}\right)}=\beta_{\left(H_{i}, V_{i}\right)}$ if $\alpha_{\left(H_{j}, V_{j}\right)}=\beta_{\left(H_{j}, V_{j}\right)}$ for any $j<i$. Thus, by induction we see that $\alpha_{(H, V)}=\beta_{(H, V)}$ for any $(H, V) \in T(G)$, or that $[M]=[N]$ in $I_{n}^{R}[G]$.

Corollary 2.7 (cf. [4]). Suppose that $M$ and $N$ are $G$-cobordant closed G-manifolds with $M^{H, 1}=\phi$ and $N^{H, 1}=\phi$ for any subgroup $H$ of $G$ 。 Then there exists a Reinhart $G$-cobordism between $M$ and $N$, if and only if $\chi\left(M^{(H, V)}\right)=\chi\left(N^{(H, V)}\right)$ for any pair $(H, V)$.

Proof. The "only if" part follows from Lemma 2.1. If $\chi\left(M^{(H, V)}\right)$ $=\chi\left(N^{(H, V)}\right)$ for any $(H, V)$, then $[M]-[N]=0$ in $\bar{I}_{n}^{R}[G]$ by Theorem 2.4 and Proposition 2.6. This implies that there exists a Reinhart $G$-cobordism between $M$ and $N$.

\section{§3. Controllable Cutting and Pasting}

As in $\S 1$ and $\S 2$ of Heithecker [2] we obtain the following Proposition 3. 1, Lemma 3.2 and Lemma 3.3:

Proposition 3. 1. If $[M]=[N]$ in $S K K_{n}[G]$, then

(i) $\quad[M]=[N]$ in $N_{n}[G]$, and

(ii) $\chi\left(M^{(H, V)}\right)=\chi\left(N^{(H, V)}\right)$ for any pair $(H, V)$.

Lemma 3.2. If $N$ is obtained from $M$ by $G$-surgery of type ( $H, V$, $p, q)$, then in $S K K_{n}[G]$,

$$
[M]+\left[G \times{ }_{H} S\left(V \oplus R^{p+q}\right)\right]=[N]+\left[G \times{ }_{H} S\left(V \oplus R^{p+1}\right) \times S\left(R^{q}\right)\right],
$$

where $p+q=n-\operatorname{dim} V+1$.

Lemma 3. 3. In $S K K_{n}[G]$, 


$$
\left[G \times{ }_{H} S\left(V \oplus R^{p+1}\right) \times S\left(R^{q}\right)\right]= \begin{cases}2\left[G \times{ }_{H} S\left(V \oplus R^{p+q}\right)\right] & \text { if } q \text { is odd, } \\ 0 & \text { if } q \text { is even, }\end{cases}
$$

where $p+q=n-\operatorname{dim} V+1$.

The following proposition follows from the preceding two lemmas.

Proposition 3.4. If $N$ is obtained by performing a finite series of $G$-sugeries on $M$ of type $\left(H_{i}, V_{i}, p_{i}, q_{i}\right), i=1,2, \ldots, s$, then in $S K K_{n}[G]$,

$$
[N]=[M]+\sum_{i=1}^{s}(-1)^{q_{i}}\left[G \times_{H_{i}} S\left(V_{i} \oplus R^{n-\mathrm{dimV}_{i}+1}\right)\right] .
$$

Let $I_{n}[G]$ be the subgroup of $S K K_{n}[G]$ generated by $G$-manifolds of the form $G \times{ }_{H} S(V)$. From Proposition 3.4 and Lemma 1.1, if $M$ and $N \in \mathscr{M}_{n}[G]$ are $G$-cobordant, then we see that $[M]-[N] \in I_{n}[G]$. Thus we obtain

Theorem 3.5. There is a short exact sequence

$$
0 \longrightarrow I_{n}[G] \stackrel{i}{\longrightarrow} S K K_{n}[G] \stackrel{j}{\longrightarrow} N_{n}[G] \longrightarrow 0
$$

where $i$ and $j$ are the obvious homomorphisms.

Proposition 3.6. If $[M]=[N]$ in $N_{n}^{R}[G]$, then $[M]=[N]$ in $S K K_{n}[G]$.

Proof. From the hypothesis there is a Reinhart $G$-cobordism $L$ between $M$ and $N$, and by Lemma 2.1 $L$ satisfies $\chi\left(L^{(H, V)}\right)=\chi\left(M^{(H, V)}\right)$ $=\chi\left(N^{(H, V)}\right)$ for any pair $(H, V)$. By Lemma $1.1, N$ is obtained from $M$ by performing a finite series of $G$-surgeries of type $\left(H_{i}, V_{i}\right.$, $\left.p_{i}, q_{i}\right), i=1,2, \ldots, s$, with trace $L$. Here we may take the subgroups $H_{1}, H_{2}, \ldots, H_{s}$ so that $H_{i}=H_{j}$ if $H_{i}$ and $H_{j}$ are conjugate. Moreover, we may take the representations $V_{1}, V_{2}, \ldots, V_{s}$ so that $V_{i}=V_{j}$ if $H=H_{i}=H_{j}$, and if $G \times_{H} V_{i}$ and $G \times{ }_{H} V_{j}$ are isomorphic as $G$-vector bundles over $G / H$. By Proposition 3. 4 in $S K K_{n}[G]$,

$$
[N]=[M]+\sum_{i=1}^{s}(-1)^{q_{i}}\left[G \times_{H_{i}} S\left(V_{i} \oplus R^{n-\operatorname{dim} V_{i}+1}\right)\right] .
$$

Divide the set $I=\{1,2, \ldots, s\}$ into the disjoint union of subsets, $I=I_{1} \cup I_{2} \cup \ldots \cup I_{a}$, such that for any $b(1 \leqq b \leqq a), i, j \in I_{b}$ if and only if $H_{i}=H_{j}$ and $V_{i}=V_{j}$. Let $H=H_{1}$ be a maximal subgroup in 
$\left\{H_{1}, H_{2}, \ldots, H_{s}\right\}$. Also let $V=V_{1}$ and $l \in I_{1}$. Note then that $H=H_{i}$ and $V=V_{i}$ for all $i \in I_{1}$. Note also that $N^{(H, V)}$ is obtained from $M^{(H, V)}$ by performing a finite series of (nonequivariant) surgeries of type $\left(\{1\},\{0\}, p_{i}, q_{i}\right), i \in I_{1}$, in which each type repeats $\chi\left(G_{(H, V)} / H\right)$ times. Since $L^{(H, V)}$ is the trace of this series of surgeries, we see

$$
\chi\left(L^{(H, V)}\right)=\chi\left(M^{(H, V)}\right)+\chi\left(G_{(H, V)} / H\right) \sum_{i \in I_{1}}(-1)^{q_{i}} 。
$$

Since $\chi\left(L^{(H, V)}\right)=\chi\left(M^{(H, V)}\right)$, then $\sum_{i \in I_{1}}(-1)^{q_{i}}=0$. Considering inductively the same as above we see that $\sum_{i \in I_{b}}(-1)^{q_{i}}=0$ for any $b(1 \leqq b \leqq a)$. This implies $[M]=[N]$ in $S K K_{n}[G]$.

Consider the following commutative diagram

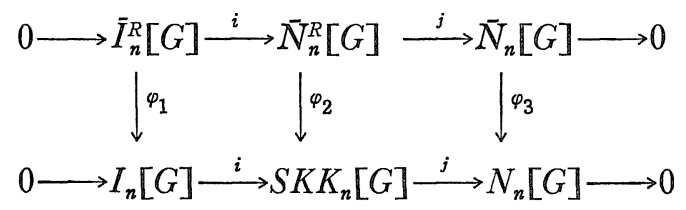

where $\varphi_{1}, \varphi_{2}$ and $\varphi_{3}$ are the obvious homomorphisms. Note that $\varphi_{1}$ and $\varphi_{2}$ are well-defined by the preceding proposition.

Proposition 3.7. $\varphi_{2}: \bar{N}_{n}^{R}[G] \rightarrow S K K_{n}[G]$ is injective.

Proof. In the above diagram the two rows are exact and $\varphi_{3}$ is injective. Thus it suffices to prove the injectivity of $\varphi_{1}$. Suppose that $[M]-[N] \in \bar{I}_{n}^{R}[G]$ and $[M]-[N]=0$ in $I_{n}[G]$. Proposition 3.1 shows that $\chi\left(M^{(H, V)}\right)=\chi\left(N^{(H, V)}\right)$ for any $(H, V)$. By Corollary 2.7 this shows that $[M]-[N]=0$ in $\bar{I}_{n}^{R}[G]$. Thus $\varphi_{1}$ is injective.

Corollary 2.7, Proposition 3.6 and Proposition 3.7 are now summarized as the following theorem.

Theorem 3. 8. Let $M$ and $N \in \mathscr{M}_{n}[G]$ be such that $M^{H, 1}=\phi$ and $N^{H, 1}=\phi$ for any subgroup $H$ of $G$. Then the following (i), (ii) and (iii) are equivalent:

(i) $\quad[M]=[N]$ in $N_{n}^{R}[G]$,

(ii) $[M]=[N]$ in $S K K_{n}[G]$,

(iii) $\quad[M]=[N]$ in $N_{n}[G]$, and $\chi\left(M^{(H, V)}\right)=\chi\left(N^{(H, V)}\right)$ 
for any $(H, V)$.

Notes. (1) The restriction $M^{H, 1}=\phi$ is caused by the following fact. Given a bounded compact manifold $N \neq \phi$ of dimension $2 n-1>2$, then there exists a $2 n$-dimensional compact connected manifold $L$ which bounds $N$ and has a prescribed Euler characteristic. If $n=1$, however $\chi(L)$ is at most 1 .

(2) If $n$ is even or if $G$ is abelian, from what we noted above Theorem 2. 4, the restrictions $M^{H, 1}=\phi$ and $N^{H, 1}=\phi$ in Theorem 3.8 are not needed.

(3) In the case where $G$ is of even order some different matters happen. For example, gluing by antipodal involution yields new fixed points, and the dimensions of fixed point sets are not congruent modulo 2 .

\section{References}

[1] Field, M. J., Handlebody decompositions for G-manifolds, Bull. Austral. Math. Soc., 25 (1982), 29-36.

[2] Heithecker, J., Äquivariantes kontrolliertes Schneiden und Kleben, Math. Ann., 217 (1975), 17-28.

[3] — Äquivarianter Bordismus mit Vektorfeld, Math. Ann., 234 (1978), 1-8.

[4] Komiya, K., Equivariant cobordism, vector fields and the Euler characteristic, $J$. Math. Soc. Japan, 38 (1986), 9-18.

[5] Prevot, K., Modifications of controllable cutting and pasting, Houston J. Math., 6 (1980), 565-575.

[6] - Cutting and pasting $Z_{p}$-manifolds, Canad. Math. Bull., 25 (1982), 13-28.

[7] , Cutting and pasting involutions, Rocky Mountain J. Math., 12 (1982), 233240.

[8] Reinhart, B. L., Cobordism and the Euler number, Topology, 2 (1963), 173-177.

[9] Wilczyński, D., Fixed point free equivariant homotopy classes, Fund. Math., 123(1984), 47-60. 